\title{
Two endpoint bounds for generalized Radon transforms in the plane
}

Jong-Guk Bak, Daniel M. Oberlin and Andreas Seeger

\section{Introduction}

The purpose of this note is to prove $L^{p} \rightarrow L^{q}$ inequalities for averaging operators in the plane (also known as generalized Radon transforms). To describe our setup let $\Omega_{L}$ and $\Omega_{R}$ be open sets in $\mathbb{R}^{2}$ and let $\mathcal{M}$ be a submanifold in $\Omega_{L} \times \Omega_{R}$ which will contain the singular support of the kernel of our operator. We assume that the projections $\mathcal{M} \rightarrow \Omega_{L}$ and $\mathcal{M} \rightarrow \Omega_{R}$ have surjective differential; thus the varieties

$$
\begin{aligned}
& \mathcal{M}_{x}=\left\{y \in \Omega_{R} ;(x, y) \in \mathcal{M}\right\} \\
& \mathcal{M}_{y}=\left\{x \in \Omega_{L} ;(x, y) \in \mathcal{M}\right\}
\end{aligned}
$$

are smooth immersed curves in $\Omega_{L}$ and $\Omega_{R}$, respectively.

Let $\chi \in C^{\infty}\left(\Omega_{L} \times \Omega_{R}\right)$ be compactly supported. We consider the operator

$$
\mathcal{R} f(x)=\int_{\mathcal{M}_{x}} \chi(x, y) f(y) d \sigma_{x}(y)
$$

where $d \sigma_{x}$ is a smooth density on $\mathcal{M}_{x}$ depending smoothly on $x \in \Omega_{L}$.

The regularity properties of $\mathcal{R}$ depend on certain finite type conditions, formulated in [15]. We recall that a vector field $V$ on $\mathcal{M}$ is of type $(1,0)$ on an open subset $U$ of $\mathcal{M}$ if for every $P \in U$ we have $V_{P} \in T_{P} \mathcal{M} \cap\left(T_{P} \Omega_{L} \times\{0\}\right)$. $V$ is of type $(0,1)$ on $U$ if $\left.V_{P} \in T_{P} \mathcal{M} \cap\left(\{0\} \times T_{P} \Omega_{R}\right\}\right)$ for every $P \in U$. The $C^{\infty}(U)$ modules of vector fields of type $(1,0)$ and $(0,1)$ on $U$ are denoted by $\mathcal{V}^{1,0}(U)$ and $\mathcal{V}^{0,1}(U)$, respectively. Since $\mathcal{M}$ is three-dimensional there is a nonvanishing one-form $\omega$ which annihilates $(1,0)$ and $(0,1)$ vectors. If $X$ and $Y$ are nonvanishing vector fields of type $(1,0)$ and $(0,1)$, respectively, then

2000 Mathematics Subject Classification: 35S30, 42B99.

Keywords: Generalized Radon transforms, averaging operators. 
the quantity $\langle\omega,[X, Y]\rangle$ is comparable to the rotational curvature introduced by Phong and Stein. In fact if $\mathcal{M}$ is given by the equation $\Phi(x, y)=0$ with $\Phi_{x} \neq 0, \Phi_{y} \neq 0$ and if we choose $X=\Phi_{x_{2}} \partial_{x_{1}}-\Phi_{x_{1}} \partial_{x_{2}}, Y=\Phi_{y_{2}} \partial_{y_{1}}-\Phi_{y_{1}} \partial_{y_{2}}$ and $\omega=\Phi_{x} d x-\Phi_{y} d y$, then $\langle\omega,[X, Y]\rangle / 2$ is equal to

$$
J=\operatorname{det}\left(\begin{array}{cc}
\Phi_{x y} & \Phi_{x}^{t} \\
\Phi_{y} & 0
\end{array}\right)
$$

the rotational curvature. The generalized Radon transform $\mathcal{R}$ is a Fourier integral operator of class $I^{-1 / 2}\left(\Omega_{L}, \Omega_{R} ; N^{*} \mathcal{M}^{\prime}\right)$ in the sense of [5], and $N^{*} \mathcal{M}^{\prime}$ is a local canonical graph if and only if $J$ does not vanish.

We now recall the notion of finite type $(\mu, \nu)$. We write $\operatorname{ad} V(W)=$ $[V, W]$ for the commutator of $V$ and $W$ and for integers $\mu \geq 1, \nu \geq 1$, we let $\mathcal{V}^{\mu, \nu}(U)$ denote the $C^{\infty}(U)$-module generated by all vector fields in $\mathcal{V}^{1,0}(U) \cup \mathcal{V}^{0,1}(U)$ and all vector fields of the form $g \operatorname{ad} V_{1} \cdots \operatorname{ad} V_{n-1}\left(V_{n}\right)$, where $g$ is smooth, $V_{i} \in \mathcal{V}^{1,0}(U) \cup \mathcal{V}^{0,1}(U)$, at most $\mu$ of the $V_{i}$ are in $\mathcal{V}^{1,0}(U)$ and at most $\nu$ of the $V_{i}$ are in $\mathcal{V}^{0,1}(U)$. We say that $\mathcal{M}$ is of type $(\mu, \nu)$ at $P$ if there is an open neighborhood $U$ and a vector field $V \in \mathcal{V}^{\mu, \nu}(U)$ so that $\left\langle\omega_{P}, V_{P}\right\rangle \neq 0$ but $\left\langle\omega_{P}, W_{P}\right\rangle=0$ for all $W \in \mathcal{V}^{\mu-1, \nu}(U) \cup \mathcal{V}^{\mu, \nu-1}(U)$. Thus type $(1,1)$ corresponds to the nondegenerate situation of nonvanishing rotational curvature.

Let $n \geq 2, m \geq 2$. Following [14] we also say that $\mathcal{M}$ satisfies a left finite type condition of degree $n$ in $U$ if $\mathcal{M}$ is of finite type $(1, k)$ for some $k$ with $k \in\{1, \ldots, n-1\}$, for every $P \in U$. We note (see [15]) that $\mathcal{M}$ satisfies this condition if only if for all $\left(x_{0}, y_{0}\right) \in \mathcal{U}$ the quantity $J\left(x_{0}, y\right)$ when restricted to the curve $\mathcal{M}_{x_{0}}$ vanishes of order at most $n-2$ at $y=y_{0}$. Likewise $\mathcal{M}$ satisfies a right finite type condition of degree $m$ in $U$ if $\mathcal{M}$ is of finite type $(j, 1)$ at $P$ for some $j \in\{1, \ldots, m-1\}$, for every $P \in U$. Again an equivalent formulation is that for all $P_{0}=\left(x_{0}, y_{0}\right) \in \mathcal{U}$ the quantity $J\left(x, y_{0}\right)$ when restricted to the curve $\mathcal{M}^{y_{0}}$ vanishes of order at most $m-2$ at $x=x_{0}$.

We now state an endpoint $L^{p} \rightarrow L^{q}$ estimate for two-sided finite type conditions. In fact a sharper statement can be obtained by working with Lorentz-spaces $L^{p, q}$; note that $L^{p} \subset L^{p, r}$, if $r \geq p$, with continuous embedding.

Theorem 1.1. Suppose that $\mathcal{M}$ satisfies a left finite type condition of degree $n$ and a right finite type condition of degree $m$.

(i) Suppose that $(1 / p, 1 / q)$ belongs to the closed trapezoid $\mathcal{T}(m, n)$ with corners $(0,0),(1,1),\left(\frac{m}{m+1}, \frac{m-1}{m+1}\right),\left(\frac{2}{n+1}, \frac{1}{n+1}\right)$. Then $\mathcal{R}$ maps $L^{p}$ boundedly to $L^{q}$. 
ii) $\mathcal{R}$ maps $L^{\frac{n+1}{2}, n+1}$ to $L^{n+1}$ and $L^{\frac{m+1}{m}}$ to $L^{\frac{m+1}{m-1}, \frac{m+1}{m}}$.

(iii) If there is a point $P$ such that $\chi(P) \neq 0$ and $\mathcal{M}$ is of type $(1, n-1)$ at $P$ then $\mathcal{R}$ does not map $L^{\frac{n+1}{2}, r}$ to $L^{n+1}$ if $r>n+1$. If there is a point $P$ such that $\chi(P) \neq 0$ and $\mathcal{M}$ is of type $(m-1,1)$ at $P$ then $\mathcal{R}$ does not map $L^{\frac{m+1}{m}}$ to $L^{\frac{m+1}{m-1}, s}$ for $s<(m+1) / m$.

\section{Remarks:}

(a) Let $\mathcal{G}(P)$ be the graph connecting $(0,0)$ and $(1,1)$ with the points $\left(\frac{\mu+1}{\mu+\nu+1}, \frac{\mu}{\mu+\nu+1}\right)$ for which $\mathcal{M}$ is of type $(\mu, \nu)$ at $P$ and suppose that $(1 / p, 1 / q)$ lies above $\mathcal{G}(P)$. Then a result in [15] states that $\mathcal{R}$ maps $L^{p}$ to $L^{q}$ provided that the cutoff function has sufficiently small support close to $P$; see also Phong-Stein [6], [7] for sharp endpoint bounds in several model cases. If $(1 / p, 1 / q)$ lies below $\mathcal{G}(P)$ and $\chi(P) \neq 0$ then $L^{p} \rightarrow L^{q}$ boundedness fails $([15])$. In the present situation this implies the following: If there is a point $P$ with $\chi(P) \neq 0$ such that $\mathcal{M}$ is of type $(1, n-1)$ and of type $(m-1,1)$ and if $\mathcal{M}$ is not of type $(\mu, \nu)$ at $P$ for all $(\mu, \nu)$ with $\left(\frac{\mu+1}{\mu+\nu+1}, \frac{\mu}{\mu+\nu+1}\right) \notin \mathcal{T}(m, n)$ then the result in part (i) of Theorem 1.1 is sharp. In particular, the $L^{(n+1) / 2, n+1} \rightarrow L^{n+1}$ estimate is best possible if $\mathcal{M}$ is of type $(1, n-1)$ and of type $(m-1,1)$ for some $m$.

(b) The sharp bounds for $p>(n+1) / 2, q=2 p$, and $p<m /(m-1)$, $1 / q=2 / p-1$ are in [14], [15]. The $L^{(n+1) / 2, n+1} \rightarrow L^{n+1}$ endpoint inequality for polynomial surfaces of the form $\mathcal{M}=\left\{(x, y): y_{2}=\right.$ $\left.x_{2}+\sum_{j+k \leq n} a_{j, k} x_{1}^{j} y_{1}^{k}\right\}$, with $a_{1, n-1} \neq 0$ was obtained by the first author in [1] based on multilinear arguments in [3], [11]; our proofs of Theorem 1.1 and Theorem 1.2 below rely on this technique as well.

(c) Let $\mathcal{M}$ be defined by a polynomial as in (b). Then $\mathcal{M}$ is of type $(\mu, \nu)$ at the origin if $a_{\mu, \nu} \neq 0$ but $a_{j, k}=0$ whenever $j \leq \mu$ and $k \leq \nu-1$ or $j \leq \mu-1, k \leq \nu$.

Our second result concerns weighted Radon transforms which incorporate the rotational curvature $J$ as an improving factor (see e.g. [16]), namely for $\gamma>0$ one defines

$$
\mathcal{R}_{\gamma} f(x)=\int_{\mathcal{M}_{x}} \chi(x, y)|J(x, y)|^{\gamma} f(y) d \sigma_{x}(y) .
$$

It is known ([15]) that $\mathcal{R}_{\gamma}$ maps $L^{2}$ into the Sobolev space $L_{1 / 2}^{2}$, provided that $\gamma>1 / 2$. By standard arguments combining Littlewood-Paley theory 
and (complex) interpolation (cf. [2]) one can see that $\mathcal{R}_{\gamma}: L^{p} \rightarrow L_{\alpha}^{p^{\prime}}$ if $\alpha \leq 2-3 / p, \gamma>1 / p^{\prime}$ and $1<p \leq 2$, in particular it maps $L^{3 / 2} \rightarrow L^{3}$ for $\gamma>1 / 3$. In various cases the endpoint bounds for $\gamma=1 / 3$ are known. If $\mathcal{M}$ is given by the equation $y_{2}=x_{2}+S\left(x_{1}, y_{1}\right)$ then $J=S_{x_{1} y_{1}}$ and for real analytic $S$ the endpoint $L^{3 / 2} \rightarrow L^{3}$ estimate can be deduced from the endpoint $L^{2}$ estimates for damped oscillatory integrals in Phong-Stein [9]. We shall prove an $L^{3 / 2} \rightarrow L^{3}$ endpoint estimate for the case where $S$ is a polynomial of degree $\leq N$, which will have the added feature that the operator norms depend only on $N$. In the translation invariant case such theorems were obtained by the second author in [10], [13]. As in [7] our operator is now globally defined (without inserting cutoff-functions) and we obtain an improved inequality using Lorentz-spaces. We note that the standard interpolation argument alluded to above does not seem to yield this estimate since one uses analytic interpolation with changing powers of $\gamma$.

Theorem 1.2. Define

$$
\mathcal{A} f\left(x_{1}, x_{2}\right)=\int_{-\infty}^{\infty}\left|\frac{\partial^{2} P}{\partial x_{1} \partial y_{1}}\right|^{1 / 3} f\left(y_{1}, x_{2}+P\left(x_{1}, y_{1}\right)\right) d y_{1}
$$

where $P$ is a polynomial in $\left(x_{1}, y_{1}\right)$ of degree at most $N$. Then there is a constant $C(N)$ (independent of the particular polynomial) so that for $3 / 2 \leq$ $r \leq 3$

$$
\|\mathcal{A} f\|_{L^{3, r}} \leq C(N)\|f\|_{L^{\frac{3}{2}, r}}
$$

for all $f \in L^{\frac{3}{2}, r}\left(\mathbb{R}^{2}\right)$.

If $\partial^{2} P /\left(\partial x_{1} \partial y_{1}\right)$ does not vanish identically then the operator $\mathcal{A}$ does not map $L^{3 / 2, r}$ to $L^{3, s}$ for any $s<r$.

In particular $\mathcal{A}$ maps $L^{3 / 2}$ to $L^{3}$.

The proof of Theorem 1.1 will be given in $\S 2$, and the proof of Theorem 1.2 in $\S 3$. We shall use the notation $\lesssim$ for inequalities involving admissible constants; here the definition of admissibility depends on the context and will be made precise in $\S 2$ and $\S 3$, respectively.

\section{Boundedness under finite type assumptions}

In this section we give a proof of the boundedness result in Theorem 1.1. It suffices to establish the $L^{\frac{n+1}{2}, n+1} \rightarrow L^{n+1}$ inequality. This also implies 
the $L^{\frac{m+1}{2}, m+1} \rightarrow L^{m+1}$ inequality for the adjoint operator $\mathcal{R}^{*}$ and thus the $L^{\frac{m+1}{m}} \rightarrow L^{\frac{m+1}{m-1}, \frac{m+1}{m}}$ inequality for $\mathcal{R}$.

By compactness arguments it suffices to prove the theorem for the case that our cutoff function $\chi$ is supported in a small neighborhood of a fixed point $P \in \mathcal{M}$; by performing translations we may assume that the coordinates vanish at $P$.

We may assume that $\mathcal{M}$ is given as

$$
\mathcal{M}=\left\{(x, y): y_{2}=G\left(x_{1}, x_{2}, y_{1}\right),\left|x_{1}\right|,\left|x_{2}\right|,\left|y_{1}\right| \leq 2\right\}
$$

where $G$ is a $C^{n+1}$ function defined on $[-2,2]^{3}$ and $G$ satisfies

$$
\begin{gathered}
G(0,0)=0, \quad G_{x_{1}}(0,0)=G_{y_{1}}(0,0)=0, \\
G_{x_{2}}(0,0)=1, \quad \frac{1}{2} \leq G_{x_{2}}\left(x, y_{1}\right) \leq 2 .
\end{gathered}
$$

We then also have for $x_{1}, x_{2}, y_{1} \in[-1,1]$

$$
y_{2}=G\left(x, y_{1}\right) \Longleftrightarrow x_{2}=H\left(y, x_{1}\right),
$$

where $H$ is defined on $[-1,1]^{3}$ and satisfies

$$
\begin{gathered}
H(0,0)=0, \quad H_{y_{1}}(0,0)=H_{x_{1}}(0,0)=0, \\
H_{y_{2}}(0,0)=1, \quad \frac{1}{2} \leq H_{y_{2}}\left(y, x_{1}\right) \leq 2 .
\end{gathered}
$$

Let $M=\max \{n+1, m+1\}$. We let $\|(G, H)\|_{C^{M}}$ be the maximum of all derivative of order at most $M$ of $G$ or $H$ in the cube $[-1,1]^{4}$ and assume that

$$
\|(G, H)\|_{C^{M}} \leq B
$$

note that $B \geq 1$.

The rotational curvature (with respect to the defining function $\Phi(x, y)=$ $y_{2}-G\left(x, y_{1}\right)$ is given by

$$
J\left(x, y_{1}\right)=\operatorname{det}\left(\begin{array}{ll}
G_{x_{1} y_{1}}\left(x, y_{1}\right) & G_{x_{1}}\left(x, y_{1}\right) \\
G_{x_{2} y_{1}}\left(x, y_{1}\right) & G_{x_{2}}\left(x, y_{1}\right)
\end{array}\right)
$$

By our finite type assumptions there are constants $a_{L}>0$ and $a_{R}>0$ so that

$$
\begin{gathered}
\min _{x} \max _{0 \leq k \leq n-2}\left|\frac{\partial^{k}}{\left(\partial y_{1}\right)^{k}} J\left(x, y_{1}\right)\right| \geq a_{L} \\
\min _{y} \max _{0 \leq j \leq m-2}\left|\frac{\partial^{j}}{\left(\partial x_{1}\right)^{j}}\left[J\left(x_{1}, H\left(y, x_{1}\right), y_{1}\right)\right]\right| \geq a_{R} ;
\end{gathered}
$$


(2.5-L) means that $\mathcal{M}$ is of type $(1, k)$ (some $k \leq n-1)$ and (2.5-R) means that $\mathcal{M}$ is of type $(j, 1)$ (some $j \leq m-1$ ), for any point under consideration, $c f$. the discussion in [15].

In what follows we choose

$$
0<\varepsilon \leq \frac{1}{4} \min \left\{((m+1) !)^{-1} B^{-m} a_{R}, 2^{-n-5} n^{-1} B^{-2} a_{L}\right\} .
$$

We define

$$
R f(x)=\int_{-\varepsilon}^{\varepsilon} \chi\left(x_{1}, x_{2}, y_{1}, G\left(x, y_{1}\right)\right) f\left(y_{1}, G\left(x, y_{1}\right)\right) d y_{1}
$$

where $\chi$ is the characteristic function of $[-\varepsilon, \varepsilon]^{4}$. Note that if $x_{1}, x_{2}, y_{1} \in$ $[-\varepsilon, \varepsilon]$ then $\left|G\left(x, y_{1}\right)\right| \leq 2 \varepsilon$.

It suffices to show that

$$
\|R f\|_{L^{n+1}} \lesssim\|f\|_{L^{\frac{n+1}{2}, n+1}}
$$

where the notation $\alpha \lesssim \beta$ means $\alpha \leq C \beta$ where $C$ depends only on $B, m, n$, $a_{L}, a_{R}$. Since $R$ is a positive operator we may assume that $f$ is nonnegative.

As in [1] we use a multilinear interpolation argument due to M. Christ [3]. In order to establish that $R$ maps $L^{\frac{n+1}{2}, n+1}$ to $L^{n+1}$ one shows the more general multilinear estimate

$$
\int \prod_{i=1}^{n+1} R f_{i}(x) d x \lesssim \prod_{i=1}^{n+1}\left\|f_{i}\right\|_{L^{\frac{n+1}{2}, n+1}}
$$

and by symmetry and real interpolation ([3]) this will follow from

$$
\int \prod_{i=1}^{n+1} R f_{i}(x) d x \lesssim\left\|f_{1}\right\|_{1} \prod_{i=2}^{n+1}\left\|f_{i}\right\|_{L^{n, 1}} .
$$

Now we use the change of variable $x_{2} \mapsto u_{2}=G\left(x_{1}, x_{2}, u_{1}\right)$ and write

$$
\begin{gathered}
\int \prod_{k=1}^{n+1} R f_{k}(x) d x=\iint \chi\left(x_{1}, x_{2}, u_{1}, G\left(x, u_{1}\right)\right) f_{1}\left(u_{1}, G\left(x, u_{1}\right)\right) \prod_{i=2}^{n+1} R f_{i}(x) d x d u_{1} \\
=\iint \chi\left(x_{1}, H\left(u, x_{1}\right), u_{1}, u_{2}\right) f_{1}\left(u_{1}, u_{2}\right) \times \\
\times \prod_{k=2}^{n+1} R f_{k}\left(x_{1}, H\left(u, x_{1}\right)\right)\left|\frac{\partial H}{\partial u_{2}}\left(u_{1}, u_{2}, x_{1}\right)\right| d u d x_{1}
\end{gathered}
$$


and, since $\left|(\partial H) /\left(\partial y_{2}\right)\right|$ is bounded by $B$, we may omit this factor. We have reduced matters to the estimate

$$
\int \prod_{k=2}^{n+1} R f_{k}\left(x_{1}, H\left(u, x_{1}\right)\right) d x_{1} \lesssim \prod_{i=2}^{n+1}\left\|f_{i}\right\|_{L^{n, 1}}
$$

for every $u$ with $\left|u_{1}\right| \leq \varepsilon,\left|u_{2}\right| \leq 2 \varepsilon$. In what follows we fix $u$. By Hölder's inequality it suffices to show

$$
\left(\int\left[R f\left(x_{1}, H\left(u, x_{1}\right)\right)\right]^{n} d x_{1}\right)^{1 / n} \lesssim\|f\|_{L^{n, 1}}
$$

By duality (2.8) is implied by

$$
\int R f(s, H(u, s)) g(s) d s \lesssim\|f\|_{L^{n, 1}\left(\mathbb{R}^{2}\right)}\|g\|_{L^{n /(n-1)}(\mathbb{R})}
$$

for any nonnegative step function $g$. The left hand side is equal to $(2.9)$

$$
\iint \chi\left(s, H(u, s), y_{1}, G\left(s, H(u, s), y_{1}\right)\right) f\left(y_{1}, G\left(s, H(u, s), y_{1}\right)\right) g(s) d y_{1} d s
$$

and we define

$$
\omega^{y_{1}, u}(s)=G\left(s, H(u, s), y_{1}\right)
$$

to change variables in this integral (after interchanging the order of integration).

Lemma 2.1. (i)

$$
\left(\omega^{y_{1}, u}\right)^{\prime}(s)=\frac{\left(y_{1}-u_{1}\right) E\left(s, u, y_{1}\right)}{G_{x_{2}}\left(s, H(u, s), u_{1}\right)}
$$

where

$$
\begin{aligned}
& E\left(s, u, y_{1}\right)= \\
& \qquad \int_{0}^{1} \operatorname{det}\left(\begin{array}{cc}
G_{x_{1} y_{1}}\left(s, H(u, s), u_{1}+\tau\left(y_{1}-u_{1}\right)\right) & G_{x_{1}}\left(s, H(u, s), u_{1}\right) \\
G_{x_{2} y_{1}}\left(s, H(u, s), u_{1}+\tau\left(y_{1}-u_{1}\right)\right) & G_{x_{2}}\left(s, H(u, s), u_{1}\right)
\end{array}\right) d \tau .
\end{aligned}
$$

(ii) Suppose that $u_{1}, y_{1}, s \in[-\varepsilon, \varepsilon],\left|u_{2}\right| \leq 2 \varepsilon$ and $y_{1} \neq u_{1}$. Then the derivative of $\omega^{y_{1}, u}$ vanishes at no more than $m-2$ points in $[-\varepsilon, \varepsilon]$. 
The elementary proof will be given below. Given $y_{1}, u$ there are intervals $I_{i}^{y_{1}, u}, i=1, \ldots, m$ with $\cup_{i=1}^{m} I_{i}^{y_{1}, u}=[-\varepsilon, \varepsilon]$ whose boundary points are measurable functions on $\left(y_{1}, u\right)$ so that $\omega^{y_{1}, u}$ has nonzero derivative in the interior of $I_{i}^{y_{1}, u}$. On each interval $I_{i}^{y_{1}, u}$ let $\omega \mapsto s_{i}^{y_{1}, u}(\omega)$ be the inverse function of $\omega^{y_{1}, u}$ and let $\widetilde{I}_{i}^{y_{1}, u}$ the image of $I_{i}^{y_{1}, u}$ under $\omega^{y_{1}, u}$. Then the integral (2.9) becomes

$$
\begin{aligned}
& \sum_{i=1}^{m} \int_{-\varepsilon}^{\varepsilon} \int_{I_{i}^{y_{1}, u}} \chi\left(s, H(u, s), y_{1}, \omega^{y_{1}, u}(s)\right) f\left(y_{1}, \omega^{y_{1}, u}(s)\right) g(s) d s d y_{1} \\
& =\sum_{i=1}^{m} \int_{-\varepsilon}^{\varepsilon} \int_{\omega \in \widetilde{I}_{i}^{y_{1}, u}} \chi\left(s_{i}^{y_{1}, u}(\omega), H\left(u, s_{i}^{y_{1}, u}(\omega)\right), y_{1}, \omega\right) f\left(y_{1}, \omega\right) g\left(s_{i}^{y_{1}, u}(\omega)\right)\left|\frac{d s_{i}^{y_{1}, u}}{d \omega}\right| d \omega d y_{1} \\
& \leq \sum_{i=1}^{m}\|f\|_{L^{n, 1}}\left\|T_{i, u}\right\|_{L^{\frac{n}{n-1}, \infty}}
\end{aligned}
$$

where

$$
T_{i, u} g\left(y_{1}, \omega\right)=\chi_{[-\varepsilon, \varepsilon]}\left(y_{1}\right) \chi_{\widetilde{I}_{i}^{y_{1}, u}}(\omega) g\left(s_{i}^{y_{1}, u}(\omega)\right) \frac{d s_{i}^{y_{1}, u}}{d \omega} .
$$

In order to finish the proof we have to show that $T_{i, u}$ maps $L^{n /(n-1)}$ to $L^{n /(n-1), \infty}$, that is

$$
\operatorname{meas}\left(\left\{\left(y_{1}, \omega\right):\left|T_{i, u} g\left(y_{1}, \omega\right)\right|>\lambda\right\}\right) \lesssim \frac{1}{\lambda^{n /(n-1)}}\|g\|_{L^{n /(n-1)}(\mathbb{R})}^{n /(n-1)} .
$$

The left hand side of (2.11) is equal to

$$
\begin{gathered}
\iint_{\substack{\left\{\left(y_{1}, s\right) \in[-\varepsilon, \varepsilon]^{2}, s \in I_{i}^{y_{1}, u}, g(s) \geq \lambda\left|\left(\omega^{y_{1}, u}\right)^{\prime}(s)\right|\right\}}}\left|\left(\omega^{y_{1}, u}\right)^{\prime}(s)\right| d y_{1} d s \\
\lesssim \int_{-\varepsilon}^{\varepsilon} \frac{|g(s)|}{\lambda} \operatorname{meas}\left(\left\{y_{1}:\left|y_{1}-u_{1}\right|\left|E\left(s, u, y_{1}\right)\right| \leq 2|g(s)| / \lambda\right\}\right) d s
\end{gathered}
$$

where we have used that $\left|G_{x_{2}}\right| \leq 2$. We now employ the following standard

Sublevel set estimate [4]. For any positive integer $\ell$ there is a constant $C_{\ell}$ such that for any interval $I \subset \mathbb{R}$, any $h \in C^{\ell}(I)$ and any $\gamma>0$ the inequality

$$
\operatorname{meas}\{x \in I:|h(x)| \leq \gamma\} \leq C_{\ell} \gamma^{1 / \ell} \inf _{x \in I}\left|h^{(\ell)}(x)\right|^{-1 / \ell}
$$

holds. 
In order to apply this we use

Lemma 2.2. For $u_{1}, s, y_{1} \in[-\varepsilon, \varepsilon],\left|u_{2}\right| \leq \varepsilon$ we have

$$
\max _{1 \leq k \leq n-1}\left|\frac{\partial^{k}}{\left(\partial y_{1}\right)^{k}}\left[\left(y_{1}-u_{1}\right) E\left(s, u, y_{1}\right)\right]\right| \geq 2^{-n-2} n^{-1} a_{L}
$$

Taking Lemma 2.2 for granted we apply the sublevel estimate for suitable $\ell \leq n-1$ and $\gamma=2|g(s)| / \lambda$ if $g(s) / \lambda \leq 1$ (otherwise estimate the size of any sublevel set by $2 \varepsilon$ ). We obtain

$$
\begin{aligned}
& \operatorname{meas}\left(\left\{y_{1} \in[-\varepsilon, \varepsilon]:\left|\left(y_{1}-u_{1}\right) E\left(s, u, y_{1}\right)\right| \leq 2|g(s)| / \lambda\right\}\right) \\
& \leq \min \left\{2 \varepsilon, \max _{1 \leq \ell \leq n-1} C_{\ell}\left(2^{n+3} n a_{L}^{-1}|g(s)| / \lambda\right)^{1 / \ell}\right\} \lesssim(|g(s)| / \lambda)^{1 /(n-1)}
\end{aligned}
$$

and thus by (2.12), (2.13)

$$
\begin{aligned}
\operatorname{meas} & \left(\left\{\left(y_{1}, \omega\right):\left|T_{i, u} g\left(y_{1}, \omega\right)\right|>\lambda\right\}\right) \leq C \int \frac{|g(s)|}{\lambda}\left(\frac{|g(s)|}{\lambda}\right)^{1 /(n-1)} d s \\
& =C \frac{1}{\lambda^{n /(n-1)}}\|g\|_{L^{n /(n-1)(\mathbb{R})}}^{n /(n-1)} .
\end{aligned}
$$

\subsection{Proof of Lemmas 2.1 and 2.2}

We need the following elementary

Sublemma. Let $g, h$ be functions having $N$ derivatives at a point $x$ and suppose that $\max _{j \leq r}\left|u^{(j)}(x)\right| \leq B_{r}, r \leq N$. Suppose that $\max _{0 \leq j \leq N-1} \mid\left(u h^{\prime}-\right.$ $\left.u^{\prime} h\right)^{(j)}(x) \mid \geq \alpha_{N}$. Then also

$$
\max _{1 \leq j \leq N}\left|h^{(j)}(x)\right| \geq 2^{-N} \alpha_{N}-B_{N}|h(x)| .
$$

Proof. By the Leibniz rule $\left(h^{\prime} u-h u^{\prime}\right)^{(k-1)}=\sum_{l=1}^{k} b_{k l} h^{(l)}-h u^{(k)}$ where the coefficients are given by $b_{k l}(x)=\left[\left(\begin{array}{c}k-1 \\ l-1\end{array}\right)-\left(\begin{array}{c}k-1 \\ l\end{array}\right)\right] u^{(k-l)}(x)$ if $1 \leq l<k$, and $b_{k k}(x)=u(x)$. Thus

$$
\begin{aligned}
\max _{1 \leq k \leq N-1} & \left|\left(h^{\prime} u-h u^{\prime}\right)^{(k-1)}\right| \\
& \leq \sup _{k} \sum_{l}\left|b_{k l}(x)\right| \max _{1 \leq j \leq N}\left|h^{(j)}(x)\right|+|h(x)| \max _{1 \leq k \leq N-1}\left|u^{(k)}(x)\right| \\
& \leq 2^{N-1} B_{N} \max _{1 \leq j \leq N}\left|h^{(j)}(x)\right|+B_{N}|h(x)|
\end{aligned}
$$

which implies the assertion. 
Proof of Lemma 2.1 Note that

$$
\left(\omega^{y_{1}, u}\right)^{\prime}(s)=G_{x_{1}}\left(s, H(u, s), y_{1}\right)+G_{x_{2}}\left(s, H(u, s), y_{1}\right) H_{x_{1}}(u, s) .
$$

The defining equation for $H$ is $x_{2}=G\left(u_{1}, H\left(x_{1}, x_{2}, u_{1}\right), x_{1}\right)$. Implicit differentiation yields that $H_{x_{1}}\left(u_{1}, G\left(x, u_{1}\right), x_{1}\right)=-\left(G_{x_{1}} / G_{x_{2}}\right)\left(x, u_{1}\right)$ or

$$
H_{x_{1}}\left(u_{1}, u_{2}, x_{1}\right)=-\frac{G_{x_{1}}\left(x_{1}, H\left(u, x_{1}\right), u_{1}\right)}{G_{x_{2}}\left(x_{1}, H\left(u, x_{1}\right), u_{1}\right)} .
$$

Thus

$$
\begin{aligned}
\left(\omega^{y_{1}, u}\right)^{\prime}(s) & =\left[\frac{1}{G_{x_{2}}\left(x, u_{1}\right)} \operatorname{det}\left(\begin{array}{cc}
G_{x_{1}}\left(x, y_{1}\right) & G_{x_{1}}\left(x, u_{1}\right) \\
G_{x_{2}}\left(x, y_{1}\right) & G_{x_{2}}\left(x, u_{1}\right)
\end{array}\right)\right]_{x=(s, H(u, s))} \\
& =\frac{\left(y_{1}-u_{1}\right) E\left(s, u, y_{1}\right)}{G_{x_{2}}\left(s, H(u, s), u_{1}\right)} .
\end{aligned}
$$

Now we prove (ii). Since $G_{x_{2}}$ does not vanish it suffices to show that

$$
\max _{0 \leq j \leq m-2}\left|\left(\frac{\partial}{\partial s}\right)^{j} E\left(s, u, y_{1}\right)\right| \geq \frac{a_{R}}{2} \text {. }
$$

We expand

$$
E\left(s, u, y_{1}\right)=E\left(s, u, u_{1}\right)+\left(y_{1}-u_{1}\right) r\left(s, u, y_{1}\right)
$$

where $E\left(s, u, u_{1}\right)=J\left(u_{1}, H(u, s), s\right)$ and

$$
\begin{aligned}
r\left(s, u, y_{1}\right)=\int_{0}^{1} \int_{0}^{1} & {\left[G_{x_{1} y_{1} y_{1}}\left(X, U_{1}\right) G_{x_{2}}\left(X, u_{1}\right)-\right.} \\
& \left.-G_{x_{2} y_{1} y_{1}}\left(X, U_{1}\right) G_{x_{1}}\left(X, u_{1}\right)\right]_{\substack{X=(s, H(u, s)) \\
U_{1}=u_{1}+\sigma \tau\left(y_{1}-u_{1}\right)}} d \sigma \tau d \tau .
\end{aligned}
$$

By assumption (2.5-R) we have

$$
\max _{0 \leq j \leq m-2}\left|\partial_{s}^{j} E\left(s, u, u_{1}\right)\right| \geq a_{R}
$$

To get a concrete upper bound for the derivatives of $r$ we need a well known fact about multiple applications of the chain rule. Namely let $v$ be $\mathbb{R}^{d}$-valued and let $\eta$ be a scalar function on the range of $\mu$, both in $C^{k}$. Then $(\eta \circ v)^{(k)}$ is a sum of at most $\prod_{i=0}^{k-1}(d+i)$ terms each of which is of the form $\xi w_{1} \cdots w_{\ell}$ where $\xi$ is a derivative of $\eta$, of order $\leq k$, the $w_{i}$ are derivatives of a component of $v$, of order at most $k$, and $\ell \leq k$. Of course more explicit formulas are known (such as the Faà di Bruno formula) but we don't need these here. Applying 
this with $d=2$ we see that a derivative of order $k$ of $s \mapsto G_{x_{1}}\left(s, H(u, s), y_{1}\right)$ can be estimated by $(k+1) ! B^{k+1}$, and a similar remark applies to the other terms in the integrand defining $r$. Thus by the Leibniz rule we have the bound $\left|\partial^{j} r /(\partial s)^{j}\right| \leq \sum_{l=0}^{j}\left(\begin{array}{l}j \\ l\end{array}\right)(l+1) ! B^{l+1}(j-l+1) ! B^{j-l+1} \leq(j+3) ! B^{j+2}$, $j \leq m-2$. Combining this with (2.16) and $\left|y_{1}-u_{1}\right| \leq 2 \varepsilon$ we see that the left hand side of (2.14) has a lower bound $a_{R}-2 \varepsilon(m+1) ! B^{m}$. Thus (2.14) follows by our choice of $\varepsilon$ in (2.6).

Proof of Lemma 2.2. First

$\frac{\partial^{k}}{\left(\partial y_{1}\right)^{k}}\left[\left(y_{1}-u_{1}\right) E\left(s, u, y_{1}\right)\right]=\frac{\partial^{k-1} E}{\left(\partial y_{1}\right)^{k-1}}\left(s, u, y_{1}\right)+\left(y_{1}-u_{1}\right) \frac{\partial^{k} E}{\left(\partial y_{1}\right)^{k}}\left(s, u, y_{1}\right)$.

Now we expand the $k$ th derivative of the integrand in (2.10) about $u_{1}$ and get $\frac{\partial^{k-1} E}{\left(\partial y_{1}\right)^{k-1}}\left(s, u, y_{1}\right)=M_{k}(s, u)+\rho_{k}\left(s, u, y_{1}\right)$ where

$$
M_{k}(s, u)=\frac{1}{k+1}\left[G_{x_{2}} \frac{\partial^{k+1} G_{x_{1}}}{\left(\partial y_{1}\right)^{k+1}}\right]_{\left(s, H(s, u), u_{1}\right)}
$$

and

$$
\begin{aligned}
\rho_{k}\left(s, u, y_{1}\right)=- & \frac{1}{k+1}\left[G_{x_{1}} \frac{\partial^{k+1} G_{x_{2}}}{\left(\partial y_{1}\right)^{k+1}}\right]_{\left(s, H(s, u), u_{1}\right)}+ \\
+ & \left(y_{1}-u_{1}\right) \int_{0}^{1} \int_{0}^{1}\left[G_{x_{2}}\left(x, u_{1}\right) \frac{\partial^{k+1} G_{x_{1}}}{\left(\partial y_{1}\right)^{k+1}}\left(x, U_{1}\right)\right. \\
& \left.-G_{x_{1}}\left(x, u_{1}\right) \frac{\partial^{k+1} G_{x_{2}}}{\left(\partial y_{1}\right)^{k+1}}\left(x, U_{1}\right)\right]_{\substack{x=(s, H(u, s)) \\
U_{1}=u_{1}+\sigma \tau\left(y_{1}-u_{1}\right)}} d \sigma \tau^{k} d \tau .
\end{aligned}
$$

Since $\left|G_{x_{1}}\right| \leq 8 \varepsilon B$ it is easy to see that $\left|\rho_{k}\left(s, u, y_{1}\right)\right| \leq 12 \varepsilon B^{2}$, moreover the term $\left|\left(y_{1}-u_{1}\right) \partial_{y_{1}}^{k} E\left(s, u, y_{1}\right)\right|$ above is bounded by $8 \varepsilon B^{2} /(k+1)$. Since $G_{x_{2}} \geq 1 / 2$ we obtain by the Sublemma that

$$
\begin{aligned}
\max _{k=0, \ldots, n-2} & \left|M_{k}(s, u)\right| \geq(n-1)^{-1} 2^{1-n} \times \\
& \times \max _{k=0, \ldots, n-2}\left|\frac{\partial^{k}}{\left(\partial y_{1}\right)^{k}}\left[G_{x_{1} y_{1}} G_{x_{2}}-G_{x_{2} y_{1}} G_{x_{1}}\right]_{\left(s, H(u, s), u_{1}\right)}\right|-B\left\|G_{x_{1}}\right\|_{\infty} \\
& \geq 2^{1-n} n^{-1} a_{L}-8 \varepsilon B^{2} .
\end{aligned}
$$

Here the $L^{\infty}$ norm of $G_{x_{1}}$ is taken over the cube $[-2 \varepsilon, 2 \varepsilon]^{4}$. We finally get

$$
\left|\frac{\partial^{k}}{\left(\partial y_{1}\right)^{k}}\left[\left(y_{1}-u_{1}\right) E\left(s, u, y_{1}\right)\right]\right| \geq 2^{-n} n^{-1} a_{L}-20 B^{2} \varepsilon
$$

and the assertion follows from our choice of $\varepsilon$ in (2.6). 
Remark. For the $L^{(n+1) / 2, n+1} \rightarrow L^{n+1}$ inequality the lower bound $a_{R}$ in (2.5-R) enters only in the definition of $\varepsilon$ in (2.6), the bounds depend on $m$ but not on $a_{R}$. Indeed the type $(m, 1)$ assumption can be replaced by an assumption of bounded multiplicity; i.e. there is $\ell \in \mathbb{N}$ so that for almost all $u$ (sufficiently small) the inverse images of the maps $s \mapsto G\left(s, H(u, s), y_{1}\right)$ have cardinality $\leq \ell$.

\subsection{Sharpness of Lorentz exponents}

It is well known that the necessary condition $1 / q \geq 2 / p-1$ follows by testing $\mathcal{R}$ on characteristic functions of small balls. We assume $1 / q=2 / p-1,1<$ $r<\infty$, and verify that $\mathcal{R}$ does not map $L^{p, r} \rightarrow L^{q, r-\varepsilon}$. Then applying this to the adjoint operator one also obtains the necessary condition $1 / q \geq 1 /(2 p)$ and also that $\mathcal{R}$ does not map $L^{p, r} \rightarrow L^{2 p, r-\varepsilon}$.

It suffices to consider $1 \leq p<2$. We assume that near the origin $\mathcal{M}$ is defined by $y_{2}=G\left(x, y_{1}\right)$ as in (2.1). For a large positive integer $\ell$ let $f \equiv f_{\ell}(y)=|y|^{-2 / p}$ for $2^{-4 \ell} \leq|y| \leq 2^{-\ell / 2}$. Then if $\left|x_{2}-H\left(0, x_{1}\right)\right| \approx 2^{-k}$ and $\ell \leq k \leq 2 \ell$ then $|\mathcal{R} f(x)| \geq c 2^{-k(1-2 / p)}$ and this happens on a set of measure $\approx 2^{-k}$. Thus if $\lambda_{\mathcal{R} f}$ denotes the distribution function of $\mathcal{R} f$ then $\lambda_{\mathcal{R} f}\left(2^{-k(1-2 / p)}\right) \gtrsim 2^{-k}$ and

$$
\begin{aligned}
\|\mathcal{R} f\|_{L^{q, s}} & \gtrsim\left(\int\left[\alpha \lambda_{\mathcal{R} f}^{\frac{1}{q}}(\alpha)\right]^{s} \frac{d \alpha}{\alpha}\right)^{1 / s} \\
& \gtrsim\left(\sum_{k=\ell}^{2 \ell}\left[c 2^{-k(1-2 / p)} \lambda_{\mathcal{R} f}^{1 / q}\left(c 2^{-k(1-2 / p)}\right)\right]^{s}\right)^{1 / s} \\
& \gtrsim\left(\sum_{k=\ell}^{2 \ell} c^{\prime} 2^{-k(1-2 / p+1 / q) s}\right)^{1 / s} \gtrsim \ell^{1 / s}
\end{aligned}
$$

if $1 / q=-1+2 / p$, and by a similar computation $\|f\|_{L^{p, r}} \lesssim \ell^{1 / r}$. Thus $\mathcal{R}$ does not map $L^{p, r} \rightarrow L^{q, s}$ if $s<r$.

\section{Polynomial Radon transforms with weights}

We now give a proof of Theorem 1.2. Fix a real-valued polynomial $P(s, t)$ of degree $\leq N$; we may assume that $\left(\partial^{2} P\right) /(\partial s \partial t)$ is not identically zero (otherwise there is nothing to prove).

In this section the notation $\alpha \lesssim \beta$ means $\alpha \leq C \beta$ where $C$ depends only on $N$. It suffices to establish the $L^{3 / 2,3} \rightarrow L^{3}$ boundedness since applying this result to the polynomial $P\left(y_{1}, x_{1}\right)$ and using duality implies the $L^{3 / 2} \rightarrow L^{3,3 / 2}$ 
boundedness and then by real interpolation the $L^{3 / 2, r} \rightarrow L^{3, r}$ boundedness for $3 / 2 \leq r \leq 3$. The sharpness assertion is proved as in the previous section (by working close to points with $\left.\left(\partial^{2} P\right) /(\partial s \partial t) \neq 0\right)$.

We use the argument of the previous section; now $G\left(x, y_{1}\right)=x_{2}+$ $P\left(x_{1}, y_{1}\right), H\left(y, x_{1}\right)=y_{2}-P\left(x_{1}, y_{1}\right)$ and $J\left(x_{1}, y_{1}\right)=\left(\partial^{2} P\right) /\left(\partial x_{1} \partial y_{1}\right)$ are globally defined. For each $s \in \mathbb{R}$, let $I_{1}^{s}, I_{2}^{s}, \ldots, I_{M(N)}^{s}$ be disjoint intervals with union $\mathbb{R}$ so that $t \mapsto \partial_{s} \partial_{t} P(s, t)$ has constant sign on the interior of each $I_{j}^{s}$. For $1 \leq j \leq M(N)$ let $U_{j}$ be the set of all $(s, t)$ such that $t \in I_{j}^{s}$ and we can choose the $I_{j}^{s}$ so that the $U_{j}$ are measurable. Let $\chi_{j}$ be the characteristic function of $U_{j}$ and define the operator $\mathcal{A}_{j}$ by

$$
\mathcal{A}_{j} f(x)=\int f\left(y_{1}, x_{2}+P\left(x_{1}, y_{1}\right)\right)\left|J\left(x_{1}, y_{1}\right)\right|^{1 / 3} \chi_{j}\left(x_{1}, y_{1}\right) d y_{1} .
$$

It is enough to prove that $\mathcal{A}_{j}$ maps $L^{3 / 2,3}$ to $L^{3}$, for any $j$. The goal is to show

$$
\int_{\mathbb{R}^{2}} \prod_{k=1}^{3} \mathcal{A}_{j} f_{k}(x) d x \lesssim \prod_{k=1}^{3}\left\|f_{k}\right\|_{L^{3 / 2,3}}
$$

and the argument in $\S 2$ reduces this to the following analogue of (2.8),

$$
\sup _{u \in \mathbb{R}^{2}}\left(\int\left|J\left(x_{1}, u_{1}\right)\right|^{1 / 3}\left|\mathcal{A}_{j} f\left(x_{1}, u_{2}-P\left(x_{1}, u_{1}\right)\right)\right|^{2} d x_{1}\right)^{1 / 2} \lesssim\|f\|_{L^{2,1}\left(\mathbb{R}^{2}\right)},
$$

or, with the measure $d \mu_{u}(s)=\left|J\left(s, u_{1}\right)\right|^{1 / 3} d s$, to

$$
\begin{aligned}
& \int\left|J\left(s, u_{1}\right)\right|^{1 / 3} \mathcal{A}_{j} f\left(s, u_{2}-P\left(s, u_{1}\right)\right) \chi_{j}\left(s, u_{1}\right) g(s) d s \\
= & \iint \chi_{j}(s, t)|J(s, t)|^{1 / 3}\left|J\left(s, u_{1}\right)\right|^{1 / 3} f\left(t, u_{2}+P(s, t)-P\left(s, u_{1}\right)\right) g(s) d s d t \\
\lesssim & \|f\|_{L^{2,1}(\mathbb{R})}\|g\|_{L^{2}(\mathbb{R}, d \mu)} .
\end{aligned}
$$

In view of the assumption that $J$ is not identically zero it is not hard to see that for every $u_{1}$ the function $s \mapsto P(s, t)-P\left(s, u_{1}\right)$ is not constant except for a finite set of values of $t$. Thus for almost all $t$ there are intervals $I_{i}^{t, u}, i=1, \ldots, N$ with $\cup_{i=1}^{N} I_{i}^{t, u}=\mathbb{R}$ whose boundary points are measurable functions on $(t, u)$ so that

$$
\omega^{t, u}(s)=u_{2}+P(s, t)-P\left(s, u_{1}\right)
$$

has nonzero derivative in the interior of $I_{i}^{t, u}$ and, as in the previous section, we denote by $\omega \mapsto s_{i}^{t, u}(\omega)$ the inverse function of $\omega^{t, u}$ on $I_{i}^{t, u}$ and let $\widetilde{I}_{i}^{t, u}$ be 
the image of $I_{i}^{t, u}$ under $\omega^{t, u}$. Let

$$
S_{i, j, u} g(t, \omega)=\left.\chi_{\widetilde{I}_{i}^{t, u}}(\omega) \frac{d s_{i}^{t, u}}{d \omega} \chi_{j}(s, t)|J(s, t)|^{1 / 3}\left|J\left(s, u_{1}\right)\right|^{1 / 3} g(s)\right|_{s=s_{i}^{t, u}(\omega)}
$$

and, arguing as in the proof of Theorem 1.1, we see that (3.1) follows from

$$
\operatorname{meas}\left(\left\{(t, \omega):\left|S_{i, j, u} g(t, \omega)\right|>\lambda\right\}\right) \lesssim \lambda^{-2} \int|g(s)|^{2}\left|J\left(s, u_{1}\right)\right|^{1 / 3} d s
$$

The left hand side of (3.2) is equal to

$$
\begin{aligned}
& \iint \quad\left|\left(\omega^{t, u}\right)^{\prime}(s)\right| d s d t \\
& \left\{(s, t): s \in I_{i}^{t, u},(s, t) \in U_{j},\right. \\
& |J(s, t)|^{1 / 3}\left|J\left(s, u_{1}\right)\right|^{1 / 3} g(s) \geq \\
& \left.\lambda\left|\left(\omega^{t, u}\right)^{\prime}(s)\right|\right\} \\
& \leq \int_{-\infty}^{\infty} \int_{\substack{\left\{t \in I_{j}^{s}: \\
|J(s, t)|^{1 / 3}\left|J\left(s, u_{1}\right)\right|^{1 / 3} g(s) \\
\geq \lambda\left|\frac{\partial P}{\partial s}(s, t)-\frac{\partial P}{\partial s}\left(s, u_{1}\right)\right|\right\}}}\left|\frac{\partial P}{\partial s}(s, t)-\frac{\partial P}{\partial s}\left(s, u_{1}\right)\right| d t d s
\end{aligned}
$$

and we have to show that the right hand side is controlled by

$$
\lambda^{-2} \int_{\mathbb{R}}|g(s)|^{2}\left|J\left(s, u_{1}\right)\right|^{1 / 3} d s,
$$

with constant only depending on $N$. This is accomplished by applying the following lemma to the inner integral in (3.3), with $p(t)=\frac{\partial P}{\partial s}(s, t)$ (which has constant sign on $I_{j}^{s}$ ).

Lemma 3.1. There is a constant $C(N)$ such that the following is true: If $p$ is a real-valued polynomial of degree $\leq N-1$ and $I$ is an interval with $p^{\prime}$ of constant sign on $I$, then for all $t_{1} \in I$ and all $B>0$ the inequality

$$
\int_{\substack{\left\{t \in I: B\left|p^{\prime}(t) p^{\prime}\left(t_{1}\right)\right|^{1 / 3} \\ \geq\left|p(t)-p\left(t_{1}\right)\right|\right\}}}\left|p(t)-p\left(t_{1}\right)\right| d t \leq C(N) B^{2}\left|p^{\prime}\left(t_{1}\right)\right|^{1 / 3}
$$

holds.

Proof. Note that the integration in (3.4) is always extended over a finite interval, thus we may assume that $I$ is finite.

We begin by observing that there is $C_{1}(N)$ such that for $0 \leq \theta \leq 1$

$$
|b-a|\left|p^{\prime}(a)\right|^{1-\theta}\left|p^{\prime}(b)\right|^{\theta} \leq C_{1}(N) \int_{[a, b]}\left|p^{\prime}(u)\right| d u .
$$


If $a=0, b=1$ this is true because the $L^{1}([0,1])$ and $L^{\infty}([0,1])$ norms are equivalent on the (finite-dimensional) space of polynomials of degree bounded by $N-2$. For other intervals $[a, b]$ an affine change of variables reduces to the case $a=0, b=1$.

Continuing the proof of the lemma, the set

$$
\left\{t \in I: B\left|p^{\prime}(t) p^{\prime}\left(t_{1}\right)\right|^{1 / 3} \geq\left|p(t)-p\left(t_{1}\right)\right|\right\}
$$

is contained in the union of two minimal subintervals $\left[t_{0}, t_{1}\right]$ and $\left[t_{1}, t_{2}\right]$ of $I$ (so that the defining inequality holds for $t=t_{0}$ and $t=t_{2}$ ). It is enough to bound the integral of $\left|p(t)-p\left(t_{1}\right)\right|$ over each of these intervals by $C_{1}(N) B^{2}\left|p^{\prime}\left(t_{1}\right)\right|^{1 / 3}$. The argument is the same in both cases, so we consider the integral over $\left[t_{0}, t_{1}\right]$. Clearly

$$
\int_{t_{0}}^{t_{1}}\left|p(t)-p\left(t_{1}\right)\right| d t \leq \int_{t_{0}}^{t_{1}} \int_{t}^{t_{1}}\left|p^{\prime}(v)\right| d v d t \leq\left(t_{1}-t_{0}\right) \int_{t_{0}}^{t_{1}}\left|p^{\prime}(v)\right| d v .
$$

We apply (3.5) with $\theta=1 / 3$ and see that the right hand side of (3.6) is dominated by

$$
C_{1}(N)\left(\int_{t_{0}}^{t_{1}}\left|p^{\prime}(v)\right| d v\right)^{2}\left|p^{\prime}\left(t_{0}\right)\right|^{-2 / 3}\left|p^{\prime}\left(t_{1}\right)\right|^{-1 / 3} \leq C_{1}(N) B^{2}\left|p^{\prime}\left(t_{1}\right)\right|^{1 / 3}
$$

where the last inequality holds since $B\left|p^{\prime}\left(t_{0}\right) p^{\prime}\left(t_{1}\right)\right|^{1 / 3} \geq\left|\int_{t_{0}}^{t_{1}} p^{\prime}(v) d v\right|$ and $p^{\prime}$ is of constant sign on $\left[t_{0}, t_{1}\right]$. The assertion follows from $(3.6),(3.7)$.

Remark. Suppose that the polynomial $P(s, t)$ is replaced by a $C^{2}$ function $S(s, t)$ with the property that for almost all $t_{1}$ the generic multiplicities of the maps $(s, t) \mapsto\left(S(s, t)-S\left(s, t_{1}\right), t\right)$ and $s \mapsto S_{s}(s, t)-S_{s}\left(s, t_{1}\right)$ are bounded by some number $\ell$ (here we say that $F: \mathbb{R}^{d} \rightarrow \mathbb{R}^{d}$ has generic multiplicity bounded by $\ell$ if $F^{-1}(y)$ has cardinality $\leq \ell$ for almost all $y \in \mathbb{R}^{n}$ ). In this case a variant of the argument used by the second author in [12] can be employed to show a slightly weaker inequality, namely that $\mathcal{A}$ is of restricted strong type $(3 / 2,3)$; i.e. it maps $L^{3 / 2,1}$ to $L^{3}$, with operator norm depending only on $\ell$. 


\section{References}

[1] BAK, J.-G., An $L^{p}-L^{q}$ estimate for Radon transforms associated to polynomials. Duke Math. J. 101 (2000), 259-269.

[2] Brenner, P., $L_{p}-L_{p^{\prime}}$ estimates for Fourier integral operators related to hyperbolic equations. Math. Z. 152 (1977), 273-286.

[3] Christ, M., On the restriction of the Fourier transform to curves: Endpoint results and the degenerate case. Trans. Amer. Math. Soc. 287 (1985), 223-238.

[4] Christ, M., Hilbert transforms along curves, I. Nilpotent groups. Ann. of Math. 122 (1985) 575-596.

[5] Hörmander, L., Fourier integral operators I. Acta Math. 127 (1971), 79-183.

[6] Phong, D. H. and Stein, E. M., Radon transforms and torsion. Int. Math. Res. Notices, appended to Duke Math. J. (1991), 49-60.

[7] Phong, D. H. And Stein, E. M., Models of degenerate Fourier integral operators and Radon transforms. Ann. of Math. 140 (1994), 703-722.

[8] Phong, D. H. And Stein, E. M., The Newton polyhedron and oscillatory integral operators. Acta Math. 179 (1997), 146-177.

[9] Phong, D. H. And Stein, E. M., Damped oscillatory integral operators with analytic phases. Adv. Math. 134 (1998), 146-177.

[10] Oberlin, D. M., Oscillatory integrals with polynomial phase. Math. Scand. 69 (1991), 45-56.

[11] Oberlin, D. M., Multilinear proofs for two theorems on circular averages. Colloq. Math 63 (1992), 187-190.

[12] Oberlin, D. M., Convolution with affine arclength measures in the plane. Proc. Amer. Math. Soc. 127 (1999), 3591-3592.

[13] Oberlin, D. M., Convolution with measures on polynomial curves, to appear in Math. Scand.

[14] Seeger, A., Degenerate Fourier integral operators in the plane. Duke Math. J. 71 (1993), 685-745.

[15] Seeger, A., Radon transforms and finite type conditions. J. Amer. Math. Soc. 11 (1998), 869-897.

[16] Sogge, C. D. And Stein, E. M., Averages of functions over hypersurfaces: smoothness of generalized Radon transforms. J. Anal. Math. 54 (1990), 165-188. 
Recibido: 7 de septiembre de 2000

Jong-Guk Bak

Department of Mathematics

Pohang University of Science and Technology, Pohang 790-784 and Korea Institute for Advanced Study, Seoul 130-012

Korea

bak@euclid.postech.ac.kr

Daniel M. Oberlin

Department of Mathematics

Florida State University

Tallahassee, FL 32306

USA

oberlin@math.fsu.edu

Andreas Seeger

Department of Mathematics

University of Wisconsin

Madison, WI 53706

USA

seeger@math.wisc.edu 